\title{
The Clinical Significance of Fibrin Monomers
}

\author{
Majed A. Refaai ${ }^{1}$ Paul Riley ${ }^{2}$ Tatsiana Mardovina ${ }^{2}$ \\ ${ }^{1}$ Department of Pathology and Laboratory Medicine, Thrombosis and \\ Hemostasis Research Laboratory, University of Rochester Medical \\ Center, University of Rochester, Rochester, New York, United States \\ 2 Diagnostica Stago, Inc., Parsippany, New Jersey, United States
}

\author{
Phoenix D. Bell ${ }^{1}$
}

Address for correspondence Majed A. Refaai, MD, Department of Pathology and Laboratory Medicine, University of Rochester Medical Center, 601 Elmwood Avenue Box no. 608, Rochester, NY 14642, United States (e-mail: m.refaai@rochester.edu).

Thromb Haemost 2018;118:1856-1866.

\begin{abstract}
Keywords

- thrombosis

- D-dimers

- venous thromboembolic event

- fibrin split products

- hyper-coagulation

Introduction Fibrin monomer (FM) concentrations reflect pro-thrombin activity and have the potential to predict thrombotic events relatively earlier than other haemostatic markers. Most often, FM are compared with D-dimer (DD) as increased DD have been documented in disseminated intravascular coagulation (DIC), deep vein thrombosis (DVT) and pulmonary embolism. Although DD have a high sensitivity and negative predictive value, their specificity is much lower depending on the assay chosen, clinical pre-test probability and patient condition. There are limited reports investigating the utility of FM in hyper-coagulable patients.

Methods We performed a literature search of FM concentrations in hyper-coagulable patients including those with DIC, acute ischaemic stroke, atrial fibrillation, acute myocardial infarction, venous thromboembolism (VTE) and cancer, as well as those who are pregnant or undergoing surgery.

Results FM were increased in patients with DIC and those with malignancy. In contrast, detection of VTE or post-operative DVT development is likely enhanced using both FM and DD concentrations. Similarly, measuring FM concentrations with other biomarker levels may be more beneficial in patients suffering an acute myocardial infarction or acute ischaemic stroke. Lastly, FM concentrations vary substantially throughout pregnancy with no definitive role of FM as of yet.

Conclusion Utilizing FM concentrations to assess hyper-coagulable patients seems promising; however, there are limitations including variations in FM cut-off values, the effect of patient medications and the timing of FM measurement relative to an acute event. Thus, further investigation is required before a true advantage for FM as a haemostatic marker can be established.
\end{abstract}

\section{Introduction}

Haemostasis is a complex physiological process of blood clot formation at the site of vessel injury to control bleeding. The haemostatic response is quick, localized and well regulated. During haemostasis, three steps occur simultaneously in a rapid order, including vasoconstriction, generation of a platelet plug (primary haemostasis) and activation of the coagulation process (secondary haemostasis), leading to fibrin clot forma-

tion. ${ }^{1}$ This insoluble fibrin forms a mesh incorporated into and around the platelet plug to strengthen and stabilize the clot.

Different laboratory tests can be used to monitor haemostatic status, including pro-thrombin time, activated partial thromboplastin time, fibrinogen, factor activity, platelet count and function, fibrin degradation products (FDPs; also known as fibrin split products) and D-dimers (DD). Historically, DD have been used as one of the indicators of thrombotic states such as venous thromboembolism (VTE). @ 2018 Georg Thieme Verlag KG
Stuttgart · New York
DOI https://doi.org/ 10.1055/s-0038-1673684. ISSN 0340-6245. 
Thrombin, which is a key protease of the coagulation system, cleaves fibrinopeptides A and B from fibrinogen, creating soluble fibrin monomers (SFMs). ${ }^{2}$ These SFMs can combine with fibrinogen and form non-covalently associated soluble fibrin monomer complexes (SFMCs). ${ }^{3}$ In the final steps, SFMCs polymerize, via factor XIIIa (FXIIIa) crosslinking, and a thrombus is formed (-Fig. 1). ${ }^{4}$ Since SFM concentration reflects thrombin activity, and their levels can be detected earlier than $\mathrm{DD},{ }^{5}$ SFM can be used as an alternative for assessing thrombosis. The role of SFM in various thrombotic states is relatively supported in the literature; however, there is no current comprehensive review of the clinical significance of SFM. Of note, for the purposes of biochemistry and laboratory measurement, SFM, FM, SF, FMC, and SFMC are indistinguishable from one another, though all abbreviations are used in different literature over the years. For simplicity, FM abbreviation will be used for all of these molecules in this review.

\section{Methods}

A literature search was conducted using the PubMed database with search terms including "fibrin monomers" or "fibrin monomer complexes" in general and with different hyper-coagulable states. A detailed summary of available studies was provided by M.A.R. and P.D.B. The list of literature was then reviewed, analysed and evaluated by all authors for inclusion in this article. Studies were deemed appropriate for this review if the relationship between FM and any hyper-coagulable state was appropriately examined, analysed and/or evaluated.

\section{Fibrin Monomer Concentration and Disseminated Intravascular Coagulation}

The diagnosis of disseminated intravascular coagulation (DIC) relies upon the patient's clinical picture and specific laboratory findings. DIC is often suspected in patients with sepsis, malignancy or unexplained bleeding/thrombosis. ${ }^{6}$ FM could potentially be used as an independent predictor of DIC. Wada et $\mathrm{al}^{7}$ analysed FM concentrations in 149 suspected DIC patients categorized into three groups: patients with non-DIC $(n=75)$, with DIC $(n=46)$ and patients with pre-DIC $(n=28)$ who developed DIC within 1 week after clinical progression. The presentation FM were significantly higher in the DIC group (mean \pm standard deviation [SD]: $363 \pm 314 \mu \mathrm{g} / \mathrm{mL}$ ) versus the pre-DIC group $(181 \pm 132 \mu \mathrm{g} / \mathrm{mL}, p<0.01)$. Nevertheless, pre-DIC patients also showed significantly higher FM versus patients with non-DIC $(52.5 \pm 50.4 \mu \mathrm{g} / \mathrm{mL}, p<0.01)$. When DIC patients were treated with gabexate mesylate (FOY), FM significantly decreased to $244 \pm 340 \mu \mathrm{g} / \mathrm{mL}(p<0.05)$. Thus, FM could be used as a marker to help determine DIC severity.

Similarly, Singh et $\mathrm{l}^{8}$ evaluated the utility of FM and DD in 70 patients with suspected DIC. Patients were separated into three groups: overt DIC $(n=32)$, non-overt DIC $(n=24)$ and non-DIC $(n=14)$. The median DD was significantly different in the overt DIC group compared with the non-overt and

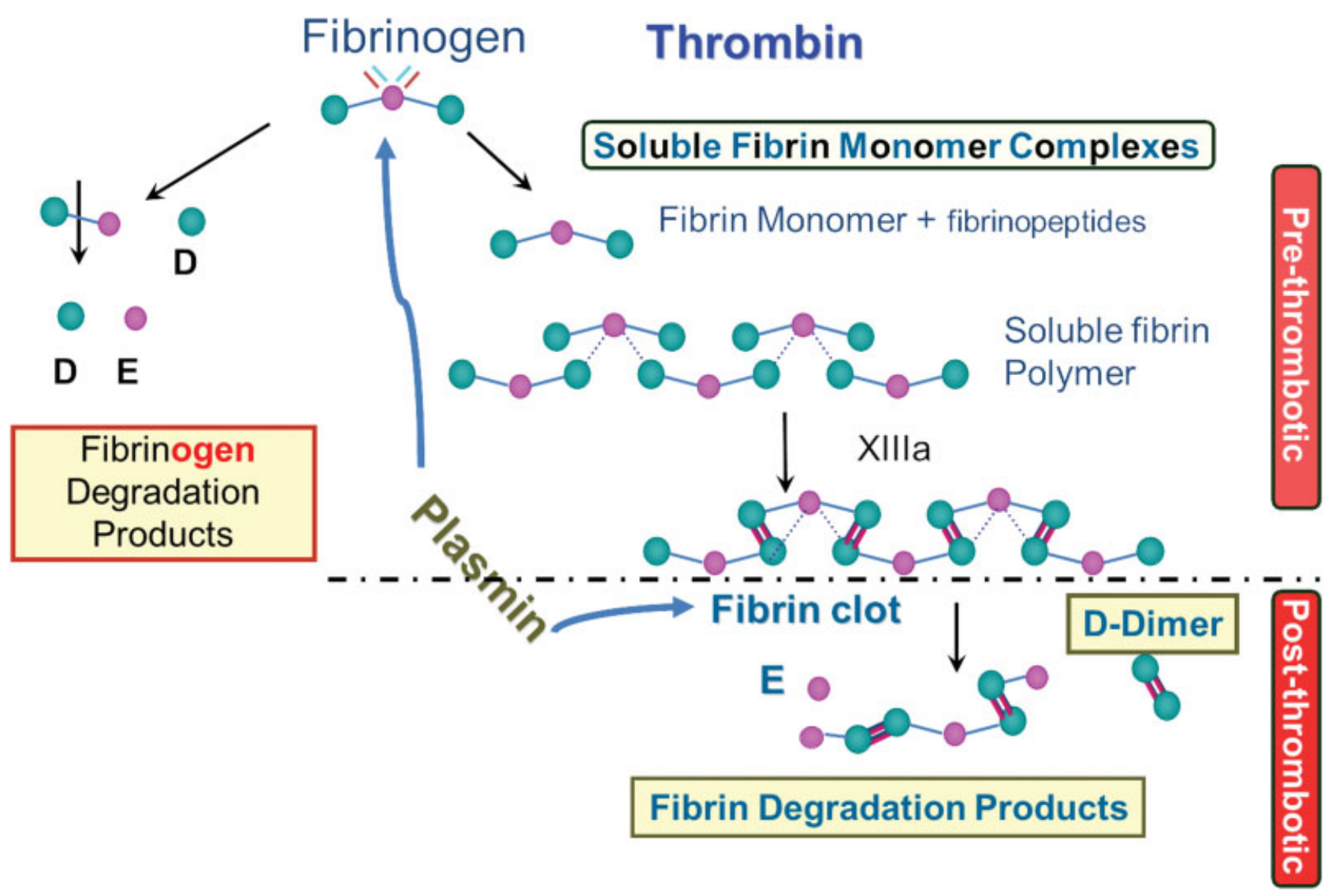

Fig. 1 Formation of soluble fibrin monomer complexes. ${ }^{4}$ 
1858 The Clinical Significance of Fibrin Monomers Refaai et al.

Table 1 Outcome summary of the included fibrin monomer studies grouped per the hyper-coagulability condition

\begin{tabular}{|c|c|c|}
\hline Authors & Type of study & Outcome \\
\hline \multicolumn{3}{|c|}{ FM and disseminated intravascular coagulation (DIC) } \\
\hline Dempfle et al ${ }^{10}$ & Prospective & FM improves the prognostic performance of the overt DIC score $(N=359)$ \\
\hline Wada et al ${ }^{7}$ & Retrospective & FM could be used to help distinguish patients with non-DIC, pre-DIC and DIC $(N=149)$ \\
\hline Singh et al ${ }^{8}$ & Prospective & FM are superior to DD in detecting DIC severity $(N=70)$ \\
\hline Selim et a $\left.\right|^{9}$ & Prospective & FM could help identify septic neonates at risk of developing DIC $(N=23)$ \\
\hline Park et al ${ }^{6}$ & Retrospective & FM is comparable to DD at predicting DIC $(N=139)$ \\
\hline Okamoto et al ${ }^{12}$ & Prospective & No appropriate cut-off value for FM exists to distinguish patients with 'pre-DIC' from those 'without DIC' $(N=613)$ \\
\hline \multicolumn{3}{|c|}{ FM and venous thromboembolism (VTE) } \\
\hline Dopsaj et al ${ }^{17}$ & Retrospective & Using both DD and FM for DVT exclusion is superior to using DD alone $(N=121)$ \\
\hline Reber et al ${ }^{19}$ & Retrospective & FM may be a useful marker inpatients with clinically suspected PE $(N=426)$ \\
\hline Schutgens et al ${ }^{16}$ & Retrospective & FM in patients with a high PTP of DVT may decreased the need for serial compression ultrasounds ( $N=464)$ \\
\hline Elias et al ${ }^{18}$ & Prospective & FM is not useful in detecting small DVTs $(N=231)$ \\
\hline \multicolumn{3}{|l|}{ FM and malignancy } \\
\hline 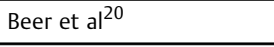 & Prospective & FM can be used to risk stratify cancer patients $(N=268)$ \\
\hline Biggerstaff et al ${ }^{21}$ & Basic science & FM enhances platelet adhesion to tumour cells and may contribute to metastasis \\
\hline Seeholzer et a ${ }^{22}$ & Retrospective & FM may be associated with tumour growth $(N=25)$ \\
\hline Tsimafeyeu et al. ${ }^{23}$ & Retrospective & $\begin{array}{l}\text { FM may be used to predict chemotherapy response and survival in patients with metastatic } \\
\text { renal cell carcinoma }(N=289)\end{array}$ \\
\hline \multicolumn{3}{|c|}{ FM and acute ischaemic stroke (AIS) } \\
\hline Hirano et al. ${ }^{27}$ & Retrospective & FM can be used to discriminate patients with cardioembolic versus non-cardioembolic stroke $(N=69)$ \\
\hline Tamura et a $2^{25}$ & Retrospective & Increased FM in AIS patients may contribute to the development of future cerebrovascular events ( $N=113$ ) \\
\hline Okuyama et $\mathrm{al}^{26}$ & Retrospective & $\begin{array}{l}\text { FM may be used to predict the presence of intra-cardiac emboli, or a hyper-coagulable state, } \\
\text { in AIS patients }(N=204)\end{array}$ \\
\hline Fassbender et al ${ }^{24}$ & Retrospective & AIS patients had increased FM levels; however, levels were even higher after rtPA administration $(N=44)$ \\
\hline \multicolumn{3}{|l|}{ FM and atrial fibrillation (AF) } \\
\hline Sato et a $2^{29}$ & Retrospective & The interaction between vWF and fibrin may promote thrombosis in AF ( $N=183)$ \\
\hline Rivera-Caravaca et al ${ }^{31}$ & Retrospective & In AF, unclear whether FM enhance risk stratification $(N=1,226)$ \\
\hline Sadanaga and Mitamura ${ }^{3}$ & Retrospective & FM do not predict future thromboembolic events in patients with $\mathrm{AF}(N=122)$ \\
\hline Tóth et a ${ }^{32}$ & Retrospective & No differences in FM were seen in patients with AF versus without AF $(N=24)$ \\
\hline \multicolumn{3}{|c|}{ FM and acute myocardial infarction (AMI) } \\
\hline leko et $\mathrm{al}^{5}$ & Retrospective & FM is a predictor of early coronary thrombus development in the first 24 hours of AMI $(N=47)$ \\
\hline Elged et al ${ }^{34}$ & Retrospective & FM could enhance AMI detection in patients with chest pain $(N=75)$ \\
\hline Kontny et al ${ }^{33}$ & Retrospective & FM could be used to predict mortality following AMI $(N=293)$ \\
\hline Brugger-Anderson et a $\left.\right|^{35}$ & Prospective & FM is not a good prognostic marker for AMI as it is influenced by $\mathrm{PCl}$ and tenecteplase treatments $(\mathrm{N}=38)$ \\
\hline \multicolumn{3}{|l|}{ FM and pregnancy } \\
\hline Van Wersch and Ubachs ${ }^{2}$ & Retrospective & $\begin{array}{l}\text { FM are elevated throughout pregnancy and may be used as a baseline comparison for high-risk } \\
\text { pregnancies associated with a hyper-coagulable state }(N=56)\end{array}$ \\
\hline Grossman et al $\left.\right|^{38}$ & Prospective & FM may enhance the detection of VTE in pregnancy $(N=2,870)$ \\
\hline Onishi et al ${ }^{36}$ & Retrospective & FM could be used to monitor hyper-coagulability in pregnancy $(N=215)$ \\
\hline Joly et al ${ }^{37}$ & Retrospective & FM are not a useful marker for hyper-coagulability throughout pregnancy, as their levels fluctuate $(N=101)$ \\
\hline \multicolumn{3}{|l|}{ FM and surgery } \\
\hline Vogel et al ${ }^{39}$ & Retrospective & FM is a potential early marker for DVT during the surgical period $(N=129)$ \\
\hline Hasegawa et al ${ }^{40}$ & Retrospective & FM may help diagnose subclinical VTE or help predict post-op VTE $(N=326)$ \\
\hline Kochi et al ${ }^{44}$ & Retrospective & FM in surgical patients could be used as an indication for anti-coagulation therapy $(N=123)$ \\
\hline Yoshioka et al ${ }^{42}$ & Retrospective & Elevated FM on POD1 in spinal surgery patients may indicate future DVT development $(N=72)$ \\
\hline Watanabe et al ${ }^{43}$ & Retrospective & FM may be an early predictor of post-op thrombosis development in spinal surgery patients $(N=56)$ \\
\hline Mitani et al ${ }^{41}$ & Retrospective & FM may be useful for early DVT diagnosis $(N=50)$ \\
\hline
\end{tabular}

Abbreviations: DD, D-dimer; DVT, deep vein thrombosis; FM, fibrin monomer; PCI, percutaneous coronary intervention; PE, pulmonary embolism; POD1, postop day 1; PTP, pre-test probability; rtPA, recombinant tissue plasminogen activator; vWF, von Willebrand factor.

Note: Studies are listed in order of clinical relevance. 
non-DIC groups ( 6.55 vs. 2.84 and $2.35 \mu \mathrm{g} / \mathrm{mL} ; p=0.001$ and $p=0.003$, respectively). However, the median DD was not significantly different between the non-overt and non-DIC groups $(p=0.17)$. In comparison, the mean FM concentration was significantly different between patients with overt DIC, non-overt DIC and non-DIC (55.6 vs. 9.65 and $5.98 \mu \mathrm{g} /$ $\mathrm{mL} ; p<0.001$ by chi-square). Furthermore, multivariate analysis showed only FM could independently differentiate patients with overt DIC from non-DIC (odds ratio [OR], 43.4; confidence interval [CI], 4.61-406.68; $p=0.001$ ), as well as patients with non-overt DIC from non-DIC (OR, 18.3; CI, 3.45-97.19; $p=0.001$ ). Ultimately, the authors proposed that FMs are superior to DD in detecting DIC severity.

Selim et $\mathrm{al}^{9}$ evaluated FM concentrations in healthy $(n=10)$ versus septic neonates $(n=13)$, of which, 10 of 13 were diagnosed with DIC. FMs were significantly higher in septic neonates without DIC versus healthy neonates $(33.69 \pm 11.85$ vs. $24.5 \pm 6.09 \mu \mathrm{g} / \mathrm{mL}, p<0.05)$. FMs were also significantly higher in septic neonates with DIC versus septic neonates without DIC $(73.2 \pm 31.55$ vs. $33.69 \pm 11.85 \mu \mathrm{g} / \mathrm{mL}$; $p<0.001$ ). The receiver operating characteristic (ROC) curve analysis established the FM cut-off at $48.5 \mu \mathrm{g} / \mathrm{mL}$, with the sensitivity for diagnosing DIC of $100 \%$, specificity of $95 \%$ and overall accuracy of $97.5 \%$. The authors suggested FM concentrations could help identify septic neonates at risk of developing DIC.

FMs were also shown to be a better predictor of mortality in early-stage DIC in critically ill adult patients. Utilizing two different DD assays, MDA (bioMerieux Inc., Durham, North Carolina, United States) and TINAquant (Roche Diagnostics, Mannheim, Germany), and Iatro FM assay (Mitsubishi Kagaku Iatron, Inc., Tokyo, Japan), Dempfle et al ${ }^{10}$ compared the use of DD and FM as the fibrin-related marker in the International Society of Thrombosis and Haemostasis overt DIC score. ${ }^{11}$ These analytes were measured in 359 intensive care unit (ICU) patients over 6 months and compared with mortality. In patients with overt DIC on day 1 of ICU admission, the calculated mortality rate using MDA DD assay was 35.5\% and TINAquant DD assay was 39.3\% ( $p=0.032$ and $p=0.012$, respectively) versus $50 \%$ using FM $(p<0.001)$. Comparing day 1 results of patients without DIC and patients with DIC, the 28 -day mortality rate was $15.6 \%$ versus $35.5 \%$ $(p=0.011)$ for MDA DD assay, $15.5 \%$ versus $39.3 \%$ $(p=0.004)$ for TINAquant DD assay and $14.0 \%$ versus $50.5 \%(p<0.001)$ for FM. This group suggests FM may improve the prognostic power of the overt DIC score.

In contrast, other groups illustrated that FM could not be used alone to predict the different stages of DIC.6,12 Okamoto et al conducted a study of 613 patients with suspected DIC and separated them into three groups based on DIC severity. ${ }^{13}$ There were 368 patients without DIC, 211 with DIC and 34 with pre-DIC (who would eventually develop DIC within 7 days of admission). As expected, FM concentrations were significantly higher in patients with pre-DIC versus without DIC (median [range]: 56.1 [12.1-151] vs. 14.1 [6.2-91.8] $\mu \mathrm{g} / \mathrm{mL}$, $p<0.05)$. However, comparing patients with DIC versus patients without DIC, ROC analysis showed a sensitivity of $91.9 \%$, specificity of $41.9 \%$ and OR of 8.199 (4.987-13.48; $p<0.001)$. Whereas comparing pre-DIC patients to patients without DIC, sensitivity decreased to $80 \%$, specificity to $41 \%$ and OR to 2.780 (1.144-6.754; $p<0.05$ ). Thus, no appropriate FM cut-off value can be established to distinguish DIC severity. Park et $\mathrm{al}^{6}$ evaluated FM and DD in 139 patients with DIC-associated diseases and separated them into three groups: group 1: nonDIC ( $n=43)$, group 2: non-overt DIC $(n=80)$ and group 3: overt DIC $(n=16)$. The median FM and DD were significantly different between each group ( $p<0.001$, each). Additionally, there was significant correlation between FM and DD versus DIC score (FM: $r=0.3975, p<0.001$; DD: $r=0.4280$, $p<0.001)$. FM and DD were significantly correlated in nonDIC and non-overt DIC patients $(r=0.5556, p<0.001)$. However, no correlation was seen between FM and DD with overt DIC patients $(p=0.104)$. ROC analysis also showed an equal effectiveness of FM and DD in diagnosing DIC. They concluded that FMs are comparable to DD at predicting DIC.

As shown, these studies demonstrated significant increases of FM in DIC patients. However, it is unclear whether FM cut-off values exist for stratifying patients based on DIC severity.

\section{Fibrin Monomer Concentration and Venous Thromboembolism}

There is significant morbidity and mortality associated with the development of VTE; nonetheless, diagnosing deep venous thrombosis (DVT) or pulmonary embolism (PE) continues to be a challenge and confirmation relies upon a multimodal approach including calculation of a clinical pre-test probability (PTP) score, DD concentrations, serial compression ultrasonographies (CUS) and a computed tomography pulmonary angiogram. ${ }^{14,15}$ Schutgens et al $^{16}$ evaluated the utility of DD and FM in 446 outpatients with suspected DVT. Patients with high PTP receiving a single CUS with a normal DD or FM had a slightly higher negative predictive value (NPV) versus patients receiving serial CUS (100 vs. 98\%). Replacing the first CUS by DD and FM decreased the NPV significantly. In patients with any PTP score, the serial CUS NPV was $97 \%$. Replacing the second CUS in this group of patients by a normal FM value showed similar NPV (97\%). They concluded that, when used alone, FMs are not as useful as the DD tests in excluding DVT. However, using FM and DD in patients with a high PTP of DVT could decrease the need for serial CUS.

Dopsaj et al $^{17}$ compared FM and DD concentrations in 96 patients with suspected DVT, grouped as DVT likely $(n=17)$ and DVT unlikely $(n=79)$. Using three different DD assays, the ROC analysis demonstrated higher area under the curve (AUC) in all of the three assays. However, AUC significantly increased when FM was included with DD $(p<0.05)$. Thus, using both DD and FM is superior to DD alone in excluding DVT. Nevertheless, in another study done by Elias et al, ${ }^{18}$ three different FM assays and one DD assay were used to evaluate patients with clinically suspected DVT $(n=231)$. Patients were categorized into three groups: confirmed DVT, DVT $\geq 4 \mathrm{~cm}$ and proximal DVT. The ROC analysis revealed higher AUC values for the DD assay $0.77(0.72-0.82)$ versus those of the FM assays (range: $0.58-0.69 ; p<0.05$ ). The 
authors concluded that DD is better than FM for detecting DVT in patients with a small DVT.

Reber et $\mathrm{al}^{19}$ assessed FM and DD concentrations in 127 patients diagnosed with PE out of 426 patients with clinically suspected PE. Using a FM cut-off of $3 \mu \mathrm{g} / \mathrm{mL}$, the sensitivity was $100 \%$ (95\% CI: $97.1-100)$, specificity was $32.8 \%$ (95\% CI: 25.7-38.1) and NPV was 100\% (95\% CI: 96.3-100). Increasing the cut-off to $4 \mu \mathrm{g} / \mathrm{mL}$, the sensitivity was $98.4 \%$ (95\% CI: 94.4-99.2), specificity was 39.1\% (95\% CI: 33.6-44.7) and NPV was 98.3\% (CI: 94.1-99.8). The authors determined that FM could exclude PE in $23 \%$ of patients with the lower cut-off and $27 \%$ with the higher cut-off, ultimately suggesting FM may be useful in patients with clinically suspected PE.

There are limited studies assessing the utility of FM in early detection of DVT or PE; however, the preceding studies suggest FM could enhance current methods used to detect VTE

\section{Fibrin Monomer Concentration and Malignancy}

Patients with an undiagnosed malignancy often present with an acute hyper-coagulable state. There is insufficient literature investigating the role of FM in early detection of cancerinduced hyper-coagulability. Beer et $\mathrm{al}^{20}$ measured haemostatic factors in 268 cancer outpatients, of which 72 were in complete remission, 55 had limited disease (regional lymph node metastasis) and 141 had extensive disease (distant metastasis). In addition to FM, DD, thrombin-anti-thrombin complex (TAT), pro-thrombin fragments $1+2\left(\mathrm{~F}_{1+2}\right)$ and fibrinopeptide A were measured in all patients. All tests were significantly elevated in patients with active disease versus patients in remission ( $p<0.001$, each) including FM (mean $\pm \mathrm{SD}, 26.6 \pm 83.6$ vs. $4.9 \pm 8.9 \mu \mathrm{g} / \mathrm{mL})$. When analysing patients with active disease, all of these tests were significantly higher in the group that died versus the group that survived ( $p=0.016$, each). Since FMs were higher in patients with active malignancy and in those who died $(40.3 \pm 23.0)$ versus patients who survived $(18.5 \pm 9.6, p<0.001)$, they concluded FM could be used to risk stratify cancer patients.

To study the effect of FM on platelet aggregation and tumour cells, fluorescently labelled platelets were added to human amelanotic malignant melanoma cells. Using confocal laser scanning microscopy, Biggerstaff et $\mathrm{al}^{21}$ observed platelet aggregation after exposure to the malignant cells over 30 minutes. Limited binding of platelets to the tumour cells was apparent. Pre-treating the tumour cells with fibrinogen did not reflect a difference in platelet binding. In contrast, pretreating tumour cells with FM demonstrated a drastic increase in platelet binding. They proposed FM enhance platelet adhesion to tumour cells and may possibly contribute to metastasis.

Seeholzer et $\mathrm{al}^{22}$ evaluated FM and response rates in 25 patients with advanced breast cancer given docetaxel and enoxaparin in a phase II clinical trial. FMs were significantly decreased in patients with partial remission $(p=0.037)$ and showed a trend towards significance in those with stable disease ( $p=0.08$ ), yet no significant difference was seen in patients with progressive disease $(p=0.48)$. The authors suggested that FM may be associated with tumour growth.

Tsimafeyeu et $\mathrm{al}^{23}$ retrospectively evaluated 289 patients with metastatic renal cell carcinoma (MRCC) who had never received prior treatment. Patients were categorized into low, intermediate and high 'extent of hyper-coagulability' based upon their fibrinogen, DD and FM levels. Patients with a greater degree of hyper-coagulability had significantly higher number of metastatic sites ( $\geq 4$ vs. $1-3 ; p=0.02$ ). On univariate analysis, hyper-coagulable patients also had significantly decreased survival compared with patients with normal coagulability (median survival: 8.9 vs. 16.3 months, $p=0.001$ ). Hyper-coagulability remained an independent predictor of survival on multivariate analysis (hazard ratio [HR], 1.63; 95\% CI: 1.5-1.76). They concluded that patients with MRCC are hyper-coagulable, and those with greater degrees of hypercoagulability have decreased overall survival.

As detailed by the above studies, cancer patients are hyper-coagulable and have increased FM levels. These levels are higher in patients with active disease versus those in remission, which may be due to FM-mediated interactions between platelets and tumour cells.

\section{Fibrin Monomer Concentration and Stroke}

Current practice for stroke diagnosis utilizes a patient's clinical presentation, head imaging, electrocardiograms and cardiac enzymes. Few studies have evaluated the application of FM in stroke patients, which involves measurement of various haemostatic factors before, during and after an acute cerebrovascular accident (CVA). Along with DD, TAT and $F_{1+2}$, FMs were measured in acute ischaemic stroke patients $(n=44)$ who presented 2 to 6 hours after symptom onset and received recombinant tissue plasminogen activator (rtPA) and heparin. ${ }^{24}$ In comparison to age- and risk factor-matched control subjects, higher FMs were detected at 1,3 and 5 hours after heparin therapy $(p<0.01)$. Levels were also compared with stroke patients treated only with heparin $(n=21)$ and FMs were significantly higher after rtPA therapy versus heparin therapy alone $(p<0.05)$. Thus, FM concentrations are elevated in patients with acute ischaemic stroke but not as significantly versus following rtPA therapy.

To identify a possible marker for use in predicting stroke recurrence, Tamura et $\mathrm{al}^{25}$ measured $\mathrm{FM}$, C-reactive protein (CRP), plasmin $\alpha 2$ inhibitor complex (PIC), DD and FDP in 113 acute stroke patients following their initial cerebrovascular event. They found patients with a recurrent stroke episode had significantly higher concentrations of FM, CRP, PIC and DD versus patients without an event ( $p<0.05$, each). A significant linear relationship between FM and these markers was detected (CRP: $R=0.310, \quad p=0.004 ; \quad$ PIC: $R=0.654$, $p<0.001 ; \quad$ DD: $R=0.724, \quad p<0.001 ; \quad$ FDP: $R=0.724$, $p<0.001$ ). On multivariate analysis, only increased FM were significantly associated with the development of a future event and/or death (HR, 1.516 per + SD increase; $95 \%$ CI: 1.042-2.180; $p=0.036$ ). They suggested elevated FM in acute ischaemic stroke patients indicate hyper-coagulability, which may contribute to the development of a future cerebrovascular event.

In another study, significantly higher FMs were detected in acute ischaemic stroke patients who developed a left atrial appendage (LAA) thrombus within 7 days of stroke $(n=24)$ versus patients without thrombus development $(n=180)$ (FM: $88 \pm 52$ vs. $14 \pm 9 \mu \mathrm{g} / \mathrm{mL}, p<0.001$ ). ${ }^{26}$ Additionally, 
FM levels were an independent predictor for LAA thrombus development (risk ratio, 2.975 per $10 \mu \mathrm{g} / \mathrm{mL}$ increase; $95 \% \mathrm{CI}$ : $1.114-4.820, p=0.021$ ). They concluded that FM may be used to predict the development of intra-cardiac emboli in acute ischaemic stroke patients.

Haemostatic markers were measured on days 1, 2, 3 and 7 of hospitalization of acute cerebral infarction patients $(n=69) .{ }^{27}$ Patients were sub-grouped based on stroke type, cardioembolic versus non-cardioembolic stroke. In addition to DD and FDP, FMs were significantly higher in patients with cardioembolic stroke on day 1 of hospitalization $(37.5 \pm 68.9$ vs. $6.6 \pm 8.6 \mu \mathrm{g} / \mathrm{mL}, p<0.01)$. Hirano et $\mathrm{al}^{27}$ summarized that biomarkers, such as FM, can be used to discriminate patients with cardioembolic versus non-cardioembolic stroke in the early stages.

Overall, these studies provide convincing evidence that FM elevations are indicative of a hyper-coagulable state during acute ischaemic stroke; however, they do not indicate their future utility in early detection of CVAs.

\section{Fibrin Monomer Concentration and Atrial Fibrillation} Patients with atrial fibrillation (AF) are hyper-coagulable and at higher risk for developing an acute embolic stroke. Clinicians evaluate this stroke risk using the $\mathrm{CHA}_{2} \mathrm{DS}_{2}$-VASc score (congestive heart failure [CHF], hypertension, age $\geq 75$ years, diabetes, prior stroke/transient ischaemic attack, vascular disease, age 65-74 years, sex category) ${ }^{28}$; however, no coagulopathic markers are incorporated into this algorithm.

Sato et $\mathrm{al}^{29}$ examined haemostatic marker levels (von Willebrand factor [vWF], DD, PIC, TAT, $\mathrm{F}_{1+2}$ and FM) in 183 patients with acute ischaemic stroke and compared these levels (at admission) between patients with and without AF. Patients were categorized into mild, moderate and severe stroke severity based on the National Institutes of Health Stroke Scale. ${ }^{30}$ FM along with vWF, DD, PIC and TAT were significantly increased in AF patients compared with levels in non-AF patients (FM: 23.19 vs. $11.83 \mu \mathrm{g} / \mathrm{mL}, p=0.043$ ); however, in AF patients, only PIC $(r=0.551 ; p=0.002)$ and FM $(r=0.449 ; p=0.003)$ levels were significantly correlated with vWF. They concluded that vWF levels may reflect a hyper-coagulable state in patients with $\mathrm{AF}$ and, although less clear, the correlation between vWF and FM may suggest an interaction between $\mathrm{vWF}$ and fibrin which may promote thrombosis in AF patients.

In a different study, 1,226 patients with $\mathrm{AF}$ taking a vitamin K antagonist (for at least 6 months) were followed for 6.5 years and the number of ischaemic strokes, adverse cardiovascular events, cardiovascular deaths and all-cause deaths were recorded. ${ }^{31}$ FMs were significantly higher in patients who suffered a composite cardiovascular event (5.3 vs. $4.7 \mu \mathrm{g} / \mathrm{mL}$, $p=0.007)$ or a cardiovascular death $(6.2$ vs. $4.8 \mu \mathrm{g} / \mathrm{mL}$, $p=0.032)$. Multivariate analysis showed patients with high FM levels ( $>12 \mu \mathrm{g} / \mathrm{mL}$ ) had an increased risk for cardiovascular events (HR, 1.72; 95\% CI: 1.31-2.26), cardiovascular mortality (HR, 2.16; 95\% CI: 1.30-3.57) and all-cause mortality (HR, 1.26; 95\% CI, 1.03-1.55). FM did not correlate to the $\mathrm{CHA}_{2} \mathrm{DS}_{2}$ VASc score ( $r$ o $=0.002, p=0.938$ ), and when evaluated concomitantly with the $\mathrm{CHA}_{2} \mathrm{DS}_{2}$-VASc score, there was no difference in their predictive performance. They concluded that, for AF patients, it is unclear whether FM enhances risk stratification.

Sadanaga and Mitamura ${ }^{3}$ conducted a post hoc analysis of a reported cohort of patients with AF. In the first group, they evaluated FM levels in 21 patients with AF before and after ( $>4$ weeks) warfarin administration. In the second group, they evaluated FM levels in $101 \mathrm{AF}$ patients on long-term warfarin. Using a FM cut-off level of $3 \mu \mathrm{g} / \mathrm{mL}$, FM levels in the first group significantly decreased after warfarin administration from 3.6 to $1.5 \mu \mathrm{g} / \mathrm{mL}(p<0.01)$. In contrast, in the second group, 9 (9\%) patients experienced a thromboembolic event; ROC analysis did not result in an optimal FM cutoff level to predict these events $(p=0.68$, AUC $=0.48$ ). Additionally, when the FM cut-off level was set to $3 \mu \mathrm{g} / \mathrm{mL}$, 26 (26\%) patients had elevated FM ( $\geq 3 \mu \mathrm{g} / \mathrm{mL}$ ) and there were 2 thromboembolic events. FM levels were not associated with the development of future thromboembolic events ( $p=0.63$ by log-rank test). They concluded that there is no utility for FM in the prediction of future thromboembolic events in patients with AF.

Tóth et $\mathrm{al}^{32}$ evaluated haemostatic markers (fibrinogen, FVIII, FXIII activity, vWF antigen, TAT, FM, plasminogen, $\alpha-2$ plasmin inhibitor, plasmin $-\alpha-{ }^{2}$ - antiplasmin complex [PAP], PAI1 activity and DD) in 24 patients with AF and a control group of 14 patients with supraventricular tachycardia undergoing transcatheter radiofrequency ablation. Levels were measured in blood samples taken from the femoral vein, left atrium and LAA before the ablation procedure. They found that FVIII and vWF were significantly elevated in the femoral vein and left atrium samples of AF patients versus controls. TAT, FM, PAP and DD were significantly elevated in both groups. They concluded that there is no difference between haemostatic and fibrinolytic markers in AF and non-AF groups and that the recorded elevations were actually due to endothelial damage from catheterization.

In general, these studies represent competing views on the role of FM in the assessment of patients with AF. Some believe that FM may assist in cardiovascular risk stratification or that FM may lead to hyper-coagulability in AF patients due to interactions with vWF, while others state that FM levels have no utility in the assessment of thrombosis in AF patients.

\section{Fibrin Monomer Concentration and Acute Myocardial Infarction}

Few reports exist investigating the relationship of DD and FM to acute myocardial infarction (AMI) development. FMs were examined in long-term outcomes of patients who developed an AMI $(n=293)$ while anti-coagulated with dalteparin (Fragmin). ${ }^{33}$ FMs were significantly higher in patients with a mortality outcome 2 days post-MI versus surviving patients (median [range]: $1.8[<0.01-73.1]$ vs. $0.4[<0.01-103.5] \mu \mathrm{g} /$ $\mathrm{mL}, p<0.001$ ). Additionally, significantly higher FMs were seen in patients with $\mathrm{CHF}$, not on dalteparin or not on thrombolytic therapy ( $p=0.016$, each). After accounting for these variables, the relationship between FM and mortality remained significant (OR, $1.9,95 \% \mathrm{CI}: 1.04-3.5, p=0.04)$. Kontny et $\mathrm{al}^{33}$ concluded that the use of FM, in combination with other risk factors, could be used to predict mortality following AMI. 
Additionally, DD and FM were significantly increased in AMI patients during the first 24 hours $(n=47)$ as compared with $>24$-hour period (FM: $14.81 \pm 25.87$ vs. $1.15 \pm 0.84$ $\mu \mathrm{g} / \mathrm{mL}, p=0.003) .^{5}$ This is in contrast to the concentrations of serum creatine kinase MB (CKMB), creatine kinase and troponin $\mathrm{T}$, which were higher after 24 hours rather than within the first 24 hours. ROC analysis demonstrated FMs were superior to DD and CKMB within the first 24 hours of AMI onset. Thus, the authors concluded that measuring FM was a better predictor of early coronary thrombus development than other biomarkers. Similarly, Elged et al ${ }^{34}$ explored the impact of FM on diagnosing AMI in conjunction with cardiac troponins. When comparing patients with AMI $(n=35)$ versus those with chest pain but no AMI $(n=40)$, FM enhanced diagnostic accuracy when used in conjunction with troponins (AUC, 0.985 ; specificity, 97.5\%; positive predictive value [PPV], 97\%) compared with when soluble cardiac troponin I (s-cTnI) (AUC, 0.903; specificity, 85\%; PPV, $84.2 \%$ ) or FM (AUC, 0.946; specificity, 90\%; PPV, 89.5\%) was used alone. Thus, adding FM to an AMI workup, in parallel with s-cTnI, could enhance AMI diagnosis.

DD and FM concentrations were measured in patients $(n=38)$ admitted with a ST-elevation myocardial infarction after percutaneous coronary intervention (PCI; $n=20$ ) or thrombolytic administration $(n=18){ }^{35}$ In addition to DD, FM increased significantly after tenecteplase (mean [range]: 3.25 [0.8-14.5] vs. 1.34 [1.6-7.44] $\mu \mathrm{g} / \mathrm{mL}, p<0.001)$. However, only FM significantly increased after PCI (4.55 [0.5-396] vs. $3.25[0.5-58.2] \mu \mathrm{g} / \mathrm{mL}, p=0.013$ ). The authors concluded that FM is not a good prognostic marker for AMI as it is influenced by common therapies such as $\mathrm{PCl}$ and tenecteplase.

Overall, these studies demonstrated increased FM concentrations in patients with AMI. However, the optimal approach could include combining these tests with other laboratory investigations to enhance diagnostic accuracy.

\section{Fibrin Monomer Concentration and Pregnancy}

Routine pre-natal care does not involve extensive evaluation of coagulation/fibrinolytic markers, unless patients have a history of DVT/PE or thrombophilia. Studies examining the relationship of haemostatic factors to thrombosis during pregnancy focus on establishing baseline concentrations in normal pregnancy, as there are active haemostatic processes occurring throughout pregnancy. In an analysis of thrombophilic markers in normal pregnancies $(n=56)$, FM, TAT, DD, plasminogen, $\alpha 2$-antiplasmin and tPA were significantly correlated with gestational age (FM correlation coefficient was $0.51 ; p=0.002$ ). Additionally, all parameters, except tPA, had average concentrations which were above the upper limit of normal reference ranges (71.1\% of FM values were above the upper limit). Specifically, FM and TAT increased with gestational age and were most pronounced in the third trimester. van Wersch and Ubachs $^{2}$ concluded that coagulation and fibrinolysis markers are elevated throughout normal pregnancy, thus some of these markers, such as FM, could be used to assess hyper-coagulability in patients with high-risk pregnancies, such as thrombosis. Onishi et $\mathrm{al}^{36}$ compared FM concentrations in 87 normal pregnancies, throughout each term, to 127 non-pregnant females and one woman who developed a post-partum DVT. No significant differences in FM were seen between early and mid-pregnancy. A small significant increase was observed in late pregnancy versus early pregnancy ( $3.95 \mathrm{vs} .3 .35 \mu \mathrm{g} / \mathrm{mL}, p<0.05$ ), yet only 2 of the 39 samples (5.2\%) during late pregnancy were greater than the $95 \% \mathrm{Cl}$ value determined by analysis of the non-pregnant females. Further, DD and FDP concentrations were significantly higher in mid- and late pregnancy versus early pregnancy $(p<0.01$, each). The patient who developed a postpartum DVT had significantly higher FM $(32.4 \mu \mathrm{g} / \mathrm{mL})$ at the time of diagnosis, with levels returning to baseline after treatment. Since FM remained relatively stable throughout pregnancy compared with other biomarkers, but increased significantly during DVT, FM could be a potential thrombotic marker for uncomplicated pregnancies.

In a study analysing $\mathrm{DD}, \mathrm{F}_{1+2}$, TAT and FM concentrations in 101 women with uncomplicated pregnancies, FM concentrations fluctuated throughout pregnancy, showing no correlation with the term of pregnancy as opposed to the other markers. ${ }^{37}$ Due to this variation, the authors postulated that FM are not a useful marker for hyper-coagulability during pregnancy. Grossman et $\mathrm{al}^{38}$ investigated the association of maternal and pregnancy factors to DD and FM concentrations in 2,870 pregnant women during the first trimester. On multivariate regression analysis, FM concentrations were influenced by mothers who were overweight, had chronic high blood pressure or had a history of cocaine abuse ( $p \leq 0.03$, each). Of note, the median FM concentration in this study was $4.3 \mu \mathrm{g} / \mathrm{mL}$ whereas the median DD concentration was $0.3 \mu \mathrm{g} / \mathrm{mL}$. Since a cut-off of $6.0 \mu \mathrm{g} / \mathrm{mL}$ of FM and $0.5 \mu \mathrm{g} / \mathrm{mL}$ of DD were used to exclude VTE in non-pregnant patients, authors concluded that these concentrations are not applicable to pregnant patients. Furthermore, FM may be used for VTE exclusion in pregnancy, with properly established reference ranges and if maternal characteristics are taken into account.

As noted, assessing hyper-coagulability in pregnancy is complex and involves utilization of coagulation tests with consideration for maternal and foetal factors. Establishing a FM cut-off for early diagnosis of hyper-coagulability in pregnancy is recommended.

\section{Fibrin Monomer Concentration and Surgery}

Managing thrombosis in surgical patients is extremely important, as shown by the requirement for DVT prophylaxis following surgery. Since many post-op patients are hypercoagulable, several studies have investigated the relationship of haemostatic factors to DVT or PE development after surgery. Vogel et $\mathrm{al}^{39}$ evaluated DD, TAT, $\mathrm{F}_{1+2}$ and FM in 129 patients who underwent abdominal surgery. In 12 patients who developed a post-op DVT, FM had the highest sensitivity (91.7\%) for diagnosing DVT versus DD, TAT and $\mathrm{F}_{1+2}(75.0,41.7$ and $33.3 \%$, respectively). Interestingly, FM and DD were higher in these patients at least 1 day before DVT symptom presentation. This group concluded that FM could be used in early diagnosis of hyper-coagulable states, such as DVT, during the surgical period. Another study investigated the relationship of FM, DD and FDP to the 
development of VTE in patients undergoing orthopaedic surgery $(n=370)$, comparing 44 patients with acute VTE to 241 patients undergoing total hip arthroplasty and 85 patients undergoing total knee arthroplasty (TKA). ${ }^{40}$ Patients were separated into four groups: without VTE, post-op DVT, sub-clinical DVT and acute VTE. FMs were significantly higher in patients with acute VTE (mean [range]: 13.65 [10.9-19.0] vs. post-op DVT 3.80 [2.804.88]), sub-clinical DVT (4.20 [3.30-5.50]) and without DVT $(2.90$ [2.50-3.80] $\mu \mathrm{g} / \mathrm{mL})$. However, FMs were not significantly increased between patients with sub-clinical DVT versus without VTE. ROC analysis of patients with acute VTE and without VTE revealed high FM values (AUC $=0.9936$, sensitivity $=97.7 \%$, specificity $=97.8 \%, \mathrm{NPV}=99.6 \%$, PPV $=96.6 \%$, OR $=1,926)$. Further, ROC analysis of patients with sub-clinical DVT and without DVT demonstrated slightly lower FM. They concluded that FM may help diagnose subclinical VTE or to predict post-op VTE. Mitani et $\mathrm{al}^{41}$ evaluated 50 patients with TKA and found FM on post-op day 1 (POD1) significantly correlated with DVT onset $(25.4 \pm 24.0$ $\mu \mathrm{g} / \mathrm{mL}, p=0.001)$. In contrast, DD correlated with DVT onset on POD3 $(8.7 \pm 5.9 \mu \mathrm{g} / \mathrm{mL}, p=0.043)$. Moreover, when DD and FM were analysed together, they enhanced the detection of DVT on POD1 and POD3 ( $p<0.05$ ). This group concluded that FM may be useful for early DVT diagnosis with improved sensitivity if measured with DD.

Evaluating FM and DD in spinal surgery patients $(n=72)$ with and without VTE development, Yoshioka et $\mathrm{al}^{42}$ found patients with VTE had significantly higher FM on POD1 versus those without VTE $(55.9 \pm 17.2$ vs. $11.1 \pm 2.89 \mu \mathrm{g} / \mathrm{mL}$, $p<0.01)$. In comparison, DD were higher in patients with VTE versus without VTE on POD7 (12.5 \pm 2.95 vs. $4.3 \pm 0.39 \mu \mathrm{g} / \mathrm{mL}, p<0.01)$. ROC analysis revealed FM was a better predictor of DVT than DD (FM POD1 AUC $=0.932$; DD POD7 AUC $=0.858$ ). They concluded elevated FM on POD1 for spinal surgery patients may suggest future DVT development. Watanabe et $\mathrm{al}^{43}$ evaluated haemostatic markers in 56 patients including 27 adolescents with idiopathic scoliosis (AIS) who underwent posterior fusion and 29 patients with lumber spinal canal stenosis (LSCS) who underwent laminectomy. For patients with LSCS, median FM concentrations were significantly increased on POD1 versus pre-operatively (3.7 vs. $2.9 \mu \mathrm{g} / \mathrm{mL}, p=0.01)$. For patients with AIS, FM were significantly higher on POD1 and POD3 versus pre-op (10.0 and 10.0 vs. $3.0 \mu \mathrm{g} / \mathrm{mL}, p=0.01$, respectively). Overall, FM concentrations were significantly higher on POD1 versus later in the post-op course, indicating FMs have the potential to surpass DD as a marker for spinal surgery post-op thrombosis.

Kochi et $\mathrm{al}^{44}$ examined the role of FM in hyper-coagulability for 123 patients undergoing gastrointestinal surgery. DD on POD7 was used as the main outcome since it was previously demonstrated to be associated with the presence of VTE. Upon analysis of surgical patients who did not receive anti-coagulant therapy after surgery, multivariate analysis revealed plasma FM levels on POD1 were strong predictors for DD elevations on POD7 (OR $=4.31,85 \% \mathrm{CI}$ : $1.10-18.30$, $p=0.03$ ). There was no significant difference in the other clinical risk factors or fibrin-related markers. These findings indicate the potential role of FM in predicting hyper-coagulability and subsequent VTE. Further, the selective administration of anti-coagulant therapy to patients with high FM could be effective for preventing VTE development. ${ }^{44}$

These studies show elevated FM levels throughout the surgical period, with some studies stating FM is superior to DD in predicting DVT development, while others suggest using FM and DD together. Thus, further studies are required to determine FM utility throughout a patient's surgical stay.

\section{Discussion}

FM concentrations reflect pro-thrombin activity and have the potential to predict thrombotic events in hyper-coagulable patients earlier than other haemostatic markers. Most often, FMs are compared with DD; however, although DDs have a high sensitivity and NPV, their specificity is much lower. Following an extensive literature search, there are limited reports investigating the utility of FM in hypercoagulable patients, including those with DIC, acute ischaemic stroke, AMI, VTE and cancer, as well as those who are pregnant or undergoing surgery (-Table $\mathbf{1}$ ).

DIC is a complex physiologic process involving hyper-coagulability and increased fibrinolysis. DDs are utilized as one of the primary laboratory tests to diagnose DIC; however, the aforementioned studies have also shown significantly elevated FM levels in patients with DIC. The difficulty in utilizing haemostatic marker concentrations arises when trying to classify patients based on the DIC severity. Notably, significant increases in FM levels are often more pronounced in overt DIC versus in patients with less severe DIC, or in those who will develop DIC. Still, significant differences in FM concentrations between patients without DIC and pre-DIC/non-overt DIC have been demonstrated, ${ }^{8}$ thus FM may be specific enough to distinguish stages of DIC. Limitations in the available literature include the varying aetiologies of DIC in each patient and the co-morbidities they may have which may ultimately enhance thrombosis, and ultimately alter FM values.

Acute ischaemic stroke patients are managed based upon head imaging and cardiac markers, and less likely upon concentrations of haemostatic factors. Due to the emergent presentation of these patients, studies have investigated the relationship of FM to acute stroke only after the acute episode has occurred. Significant increases in FM have been documented in stroke patients who have had a recurrence, as well as in patients with a cardioembolic source. Additionally, medications administered during an acute stroke, such as heparin or rtPA, seem to affect FM concentrations. Future considerations could include prospectively measuring FM levels in patients at higher risk of stroke development, so as to record and compare levels prior to, and after, an event. Other potential ideas include determining whether stroke risk stratification creates groups of patients with different FM levels, how different pharmacologics influence FM levels or how co-morbidities may cause higher 'baseline' FM levels. Further studies are required to assess the value of FM concentrations in acute ischaemic stroke patients.

AF patients are known to be hyper-coagulable; however, evaluation of haemostatic biomarkers is not considered in 
current practices. In the few studies that are available, some results have shown that FM do not differ between patients with and without $\mathrm{AF}^{32}$ and $\mathrm{FM}$ are not a good predictor of future thromboembolic events, ${ }^{3}$ while others suggest FM could be related to thrombosis, ${ }^{29}$ yet it is unclear whether they could help with risk stratification. ${ }^{45}$ These controversial findings are due to numerous variables that should be considered during FM evaluation, such as prior history of a thromboembolic event, anti-coagulation prophylactic therapy and recent invasive procedures.

AMI patients are evaluated using electrocardiographies and cardiac troponins, without routine assessment of FM or DD levels. Similar to acute ischaemic stroke, studies looking at FM levels in these patients focused on concentrations following the event. Results have shown increased FM in the first 24 hours post-MI, as well as enhanced detection of AMI when used simultaneously with cardiac troponins. Thus, rather than using FM to predict AMI, FM could be used to monitor patients after clinical presentation to assess treatment response. As mentioned, literature with inquiry into FM levels before and after the event would be beneficial, as well as measuring levels more often throughout an acute event to see if there are changes hourly, for example. Additionally, research could be conducted on how patients with a history of MI may have different FM levels compared with the normal population or how patients with cardiovascular risk factors are likely to be on specific medications affecting coagulation and likely FM levels.

VTE are frequently encountered in the acute hospital setting with DD concentrations helping guide treatment. However, the role of FM levels in this setting is not well understood. From the abovementioned literature, diagnosing DVT appears optimal when both FM and DD concentrations are used together versus independently. ${ }^{16,17}$ There are less data for FM concentrations in patients with PE, although some results have demonstrated that FM can help exclude PE in patients with chest pain. ${ }^{19}$ It is still unclear whether including FM in routine testing for DVT or PE improves patient outcome. Analysis of other factors affecting FM is needed, including further studies with PE patients, studies looking at how FM levels change in DVT patients from initial presentation, to after therapy, and to the day (or days) after admission.

Hyper-coagulability is common in cancer patients, yet there has been little investigation into their FM levels. Higher FM concentrations were reported in patients with active malignancy and in those who died from their disease. ${ }^{20}$ Further, increased FM concentrations were associated with increased platelet binding, which were hypothesized to propagate metastasis. ${ }^{21}$ Cancer patients often have multiple co-morbidities, which may enhance hyper-coagulability and the difficulty in interpreting the significance of haemostatic parameters. Further studies are also required to assess the FM value in cancer patients. These studies could include looking at the level of $\mathrm{FM} /$ haemostatic markers in different cancer types and in different age groups, in addition to the type of treatment given (chemotherapy/radiation) or the length of remission.
Numerous physiologic changes occur during pregnancy and these variations are reflected in FM concentrations. Some groups have reported no correlation of FM concentration to the stage of pregnancy, ${ }^{37}$ whereas others have reported increased concentrations in advanced pregnancies or in patients with different maternal characteristics. ${ }^{38}$ Onishi et $\mathrm{al}^{36}$ reported no difference in FM concentrations between nonpregnant females and pregnant individuals. These contradictory results confirm the difficulty in assessing hyper-coagulability in pregnancy and in determining a cut-off value for FM levels, which should be considered in future studies. Research could explore FM levels after pregnancy to see if giving birth decreases FM levels immediately or if it takes some time. Perhaps mothers with elevated FM levels after birth are at higher risk of future thrombotic events. Other interesting areas could involve comparing FM levels during pregnancy and then while the mother is having natural birth versus C-section, or measuring FM levels in higher risk pregnancies, which could potentially help identify mothers who could be more at risk for perinatal complications.

Understanding the role of FM in surgical patients is essential for the investigation of post-op DVT development. Literature has shown that FM can be used to detect hypercoagulable states during the surgical period, for example, increased FM on POD1 was associated with DVT development. ${ }^{42,43}$ Similar to VTE, these studies have also shown using FM and DD together is more accurate for diagnosing early post-op DVTs. More research is needed comparing FM levels prior to, during and after surgery, as well as how DVT prophylaxis during the surgical period (whether anti-coagulants or compression stockings) could affect FM. Other ideas include comparing FM post-operatively in patients who lost blood or received blood products during the procedure, as these patients may be at higher risk of thrombosis.

In summary, utilizing FM to assess hyper-coagulable patients seems promising; however, there are limitations which require further investigation before a true advantage for this marker can be established. Each research team has used a different cut-off value for FM level to be considered 'elevated', thus without a universal cut-off value the statistical results cannot be easily compared. In addition, much of the literature has assessed FM after the initial thrombotic event, such as AMI or acute ischaemic stroke, therefore it is unknown what FM concentrations are leading up to the acute event and how they relate to levels after the event. The cutoff value is likely going to vary depending upon the cause of thrombosis, when FM levels are measured throughout a hyper-coagulable event, and if/when anti-coagulant medications are given, elements which may only be deciphered with more research.

\section{Authors' Contributions}

M.A.R.: Developing the concept of the review, editing drafts of the manuscript and revising for proper clinical content. P.R.: Revising and editing final drafts of the manuscript. T.M.: Revising and editing final drafts of the manuscript. P.D.B.: Performing the literature review, writing and editing drafts of the manuscript. 


\section{Conflict of Interest}

M.A.R. provides external consulting and professional services to Diagnostica Stago; P.R. and T.M. are salaried employees of Diagnostica Stago, Inc.. P.D.B.: None.

\section{References}

1 Lee MS, Kong J. Heparin: physiology, pharmacology, and clinical application. Rev Cardiovasc Med 2015;16(03):189-199

2 van Wersch JWJ, Ubachs JMH. Blood coagulation and fibrinolysis during normal pregnancy. Eur J Clin Chem Clin Biochem 1991;29 (01):45-50

3 Sadanaga T, Mitamura H. Soluble fibrin monomer complex levels during oral anticoagulant therapy do not predict subsequent thromboembolic events in patients with permanent atrial fibrillation. Int J Cardiol 2013;168(01):578-580

4 Wada H, Sakuragawa N. Are fibrin-related markers useful for the diagnosis of thrombosis? Semin Thromb Hemost 2008;34(01): 33-38

5 Ieko M, Naito S, Yoshida M, et al. Plasma soluble fibrin monomer complex as a marker of coronary thrombotic events in patients with acute myocardial infarction. Tohoku J Exp Med 2009;219 (01):25-31

6 Park K-J, Kwon E-H, Kim H-J, Kim S-H. Evaluation of the diagnostic performance of fibrin monomer in disseminated intravascular coagulation. Korean J Lab Med 2011;31(03):143-147

7 Wada H, Wakita Y, Nakase T, et al. Increased plasma-soluble fibrin monomer levels in patients with disseminated intravascular coagulation. Am J Hematol 1996;51(04):255-260

8 Singh N, Pati HP, Tyagi S, Upadhyay AD, Saxena R. Evaluation of the diagnostic performance of fibrin monomer in comparison to ddimer in patients with overt and nonovert disseminated intravascular coagulation. Clin Appl Thromb Hemost 2017;23(05): 460-465

9 Selim TE, Ghoneim HR, Khashaba MT, Rakha SA. Plasma soluble fibrin monomer complex is a useful predictor of disseminated intravascular coagulation in neonatal sepsis. Haematologica 2005;90(03):419-421

10 Dempfle C-E, Wurst M, Smolinski M, et al. Use of soluble fibrin antigen instead of D-dimer as fibrin-related marker may enhance the prognostic power of the ISTH overt DIC score. Thromb Haemost 2004;91(04):812-818

11 Taylor FB Jr, Toh CH, Hoots WK, Wada H, Levi M; Scientific Subcommittee on Disseminated Intravascular Coagulation (DIC) of the International Society on Thrombosis and Haemostasis (ISTH). Towards definition, clinical and laboratory criteria, and a scoring system for disseminated intravascular coagulation. Thromb Haemost 2001;86(05):1327-1330

12 Okamoto K, Wada H, Hatada T, et al; Japanese Society of Thrombosis Hemostasis/DIC subcommittee. Frequency and hemostatic abnormalities in pre-DIC patients. Thromb Res 2010;126(01): 74-78

13 Kobayashi N, Maekawa T, Takada M, Tanaka H, Gonmori H. Criteria for diagnosis of DIC based on the analysis of clinical and laboratory findings in 345 DIC patients collected by the Research Committee on DIC in Japan. Bibl Haematol 1983;(49): 265-275

14 Subramaniam RM, Snyder B, Heath R, Tawse F, Sleigh J. Diagnosis of lower limb deep venous thrombosis in emergency department patients: performance of Hamilton and modified Wells scores. Ann Emerg Med 2006;48(06):678-685

15 Wells PS, Anderson DR, Bormanis J, et al. Value of assessment of pretest probability of deep-vein thrombosis in clinical management. Lancet 1997;350(9094):1795-1798

16 Schutgens REG, Haas FJLM, Agterof MJ, Vos M, Biesma DH. The role of fibrin monomers in optimizing the diagnostic work-up of deep vein thrombosis. Thromb Haemost 2007;97(05):807-813
17 Dopsaj V, Bogavac-Stanojevic N, Vasic D, et al. Excluding deep venous thrombosis in symptomatic outpatients: is fibrin monomer aid to D-dimer analysis? Blood Coagul Fibrinolysis 2009;20 (07):546-551

18 Elias A, Cazanave G, Nguyen F, Elias M, Bongard V, Cambus JP. Comparison of the diagnostic performance of three soluble fibrin monomer tests and a D-dimer assay in patients with clinically suspected deep vein thrombosis of the lower limbs. Haematologica 2004;89(04):499-501

19 Reber G, Bounameaux H, Perrier A, de Moerloose P. Performances of the fibrin monomer test for the exclusion of pulmonary embolism in symptomatic outpatients. Thromb Haemost 1999; 81(02):221-223

20 Beer JH, Haeberli A, Vogt A, et al. Coagulation markers predict survival in cancer patients. Thromb Haemost 2002;88(05): 745-749

21 Biggerstaff JP, Seth NB, Meyer TV, Amirkhosravi A, Francis JL. Fibrin monomer increases platelet adherence to tumor cells in a flowing system: a possible role in metastasis? Thromb Res 1998; 92(06, Suppl 2):S53-S58

22 Seeholzer N, Thürlimann B, Köberle D, Hess D, Korte W. Combining chemotherapy and low-molecular-weight heparin for the treatment of advanced breast cancer: results on clinical response, transforming growth factor-beta 1 and fibrin monomer in a phase II study. Blood Coagul Fibrinolysis 2007;18(05):415-423

23 Tsimafeyeu IV, Demidov LV, Madzhuga AV, Somonova OV, Yelizarova AL. Hypercoagulability as a prognostic factor for survival in patients with metastatic renal cell carcinoma. J Exp Clin Cancer Res 2009;28(01):30-30

24 Fassbender K, Dempfle CE, Mielke O, et al. Changes in coagulation and fibrinolysis markers in acute ischemic stroke treated with recombinant tissue plasminogen activator. Stroke 1999;30(10): 2101-2104

25 Tamura H, Hirono O, Okuyama H, et al. Elevated serum fibrinmonomer levels are associated with high long-term cerebrovascular event rates in acute ischemic stroke patients. Circ J 2007;71 (10):1573-1579

26 Okuyama H, Hirono O, Liu L, Takeishi Y, Kayama T, Kubota I. Higher levels of serum fibrin-monomer reflect hypercoagulable state and thrombus formation in the left atrial appendage in patients with acute ischemic stroke. Circ J 2006;70(08):971-976

27 Hirano K, Takashima S, Dougu N, et al. Study of hemostatic biomarkers in acute ischemic stroke by clinical subtype. J Stroke Cerebrovasc Dis 2012;21(05):404-410

28 Lip GY, Nieuwlaat R, Pisters R, Lane DA, Crijns HJ. Refining clinical risk stratification for predicting stroke and thromboembolism in atrial fibrillation using a novel risk factor-based approach: the euro heart survey on atrial fibrillation. Chest 2010;137(02): 263-272

29 Sato M, Suzuki A, Nagata K, Uchiyama S. Increased von Willebrand factor in acute stroke patients with atrial fibrillation. J Stroke Cerebrovasc Dis 2006;15(01):1-7

30 Schlegel D, Kolb SJ, Luciano JM, et al. Utility of the NIH Stroke Scale as a predictor of hospital disposition. Stroke 2003;34(01): $134-137$

31 Rivera-Caravaca JM, Roldán V, Romera M, et al. Soluble fibrin monomer complex and prediction of cardiovascular events in atrial fibrillation: the observational Murcia Atrial Fibrillation Project. J Gen Intern Med 2018;33(06):847-854

32 Tóth NK, Csanádi Z, Hajas O, et al. Intracardiac hemostasis and fibrinolysis parameters in patients with atrial fibrillation. BioMed Res Int 2017;2017:3678017

33 Kontny F, Dempfle CE, Abildgaard U. Fibrin monomer antigen: a novel marker of mortality in acute myocardial infarction. Eur Heart J 1999;20(11):808-812

34 Elged AAE, El-Gamal RA, Bastawy S, Moselhy MS. Soluble fibrin monomer complex assay enhances early and accurate diagnosis of acute myocardial infarction. Int J Clin Exp Pathol 2016;9(05):9 
35 Brügger-Andersen T, Hetland $\emptyset$, Pönitz V, Grundt H, Nilsen DWT. The effect of primary percutaneous coronary intervention as compared to tenecteplase on myeloperoxidase, pregnancy-associated plasma protein A, soluble fibrin and D-dimer in acute myocardial infarction. Thromb Res 2007;119(04): 415-421

36 Onishi H, Kaniyu K, Iwashita M, Tanaka A, Watanabe T. Fibrin monomer complex in normal pregnant women: a potential thrombotic marker in pregnancy. Ann Clin Biochem 2007;44(Pt 5):449-454

37 Joly B, Barbay V, Borg J-Y, Le Cam-Duchez V. Comparison of markers of coagulation activation and thrombin generation test in uncomplicated pregnancies. Thromb Res 2013;132(03): 386-391

38 Grossman KB, Arya R, Peixoto AB, Akolekar R, Staboulidou I, Nicolaides $\mathrm{KH}$. Maternal and pregnancy characteristics affect plasma fibrin monomer complexes and D-dimer reference ranges for venous thromboembolism in pregnancy. Am J Obstet Gynecol 2016;215(04):466.e1-466.e8

39 Vogel G, Dempfle C-E, Spannagl M, Leskopf W. The value of quantitative fibrin monomer determination in the early diagnosis of postoperative deep vein thrombosis. Thromb Res 1996;81(02): 241-251
40 Hasegawa M, Wada H, Miyazaki S, et al. The evaluation of fibrinrelated markers for diagnosing or predicting acute or subclinical venous thromboembolism in patients undergoing major orthopedic surgery. Clin Appl Thromb Hemost 2018;24(01):107-114

41 Mitani G, Takagaki T, Hamahashi K, et al. Associations between venous thromboembolism onset, D-dimer, and soluble fibrin monomer complex after total knee arthroplasty. J Orthop Surg 2015;10:172

42 Yoshioka K, Kitajima I, Kabata T, et al. Venous thromboembolism after spine surgery: changes of the fibrin monomer complex and D-dimer level during the perioperative period. J Neurosurg Spine 2010;13(05):594-599

43 Watanabe $\mathrm{H}$, Inoue $\mathrm{H}$, Shiraishi $\mathrm{Y}$, et al. Difference in the changings of blood coagulation fibrinolysis markers before and after spinal surgery in adolescent and elderly patients. Int J Orthod 2017;4(02):5

44 Kochi M, Shimomura M, Hinoi T, et al. possible role of soluble fibrin monomer complex after gastroenterological surgery. World J Gastroenterol 2017;23(12):2209-2216

45 Samuelson Bannow BT. Capsule commentary on Rivera-Caravaca et al., soluble fibrin monomer complex and prediction of cardiovascular events in atrial fibrillation: the observational Murcia Atrial Fibrillation Project. J Gen Intern Med 2018;33(06):945 